\title{
Optical Properties and Radiation Stability of Magnesium Silicate
}

\author{
V.V. Harutyunyan, A.O. Badalyan \\ A.I. Alikhanyan National Scientific Laboratory, Alikhaniyan Bros. Str., \\ Yerevan, 375036, Armenia \\ Email: vharut@yerphi.am
}

(Received: July 20, 2021; Revised August 19, 2021; Accepted: August 30, 2021)

\begin{abstract}
A microwave (MW) method of hydrothermal synthesis of magnesium silicate is developed. At low temperature $\left(240{ }^{\circ} \mathrm{C}\right)$, nuclei of crystalline phase of магний silicate are obtained. Some optical characteristics of the synthesized product are determined. Based on, X-ray and thermographic studies are revealed that the synthesis of magnesium silicate from water-soluble salts of initial components in microwave ovens promotes the formation of magnesium at lower temperatures. MW synthesis of magnesium silicate ensures obtaining of nanosized powder after heat treatment at $850{ }^{\circ} \mathrm{C}$.
\end{abstract}

Keywords: hydrothermal-microwave treatment, magnesium silicate, thermoregulating coatings, electron irradiation, diffraction, diffusion reflection, photoluminescence

https://doi.org/10.52853/18291171-2021.14.3-132

\section{Introduction}

Silicates of the second group elements have a very diverse application: from construction to precision technologies. They are widely distributed in the form of various minerals however for special purposes they are often required in the pure state and without impurities. Pigments and ceramics based on magnesium silicates, due to their valuable properties, are widely used as absorbers, decolouring agents, catalysts, fillers for paints, in the production of glass, ceramics, for thin-layer chromatographic separation of mixtures of substances extracted from biological objects. The use of magnesium silicate in aviation and space technology as pigments for thermoregulating coatings for aircraft and space vehicles is promising due to their high reflectivity in the visible spectrum of solar radiation as well as excellent radiation resistance.

The main methods for producing ceramics and ceramic pigments are as follows: calcining a mixture of natural raw materials (talc, wollastonite, tripoli, quartz, etc.), the joint fusion of salts or hydroxides of the corresponding metals, followed by calcining the mixture, as well as sol-gel methods [1-7].

An urgent task is the development of new promising methods of obtaining heat-stable radiation-resistant materials for use in space materials science. Over time, interest in chemical reactions proceeding under microwave heating conditions is growing [8-17]. Microwave treatment combined with hydrothermal processing is an effective way of obtaining inorganic materials due to uniform and fast heating, control over the processing time, and high purity conditions of the process. The hydrothermal microwave (HTMW) synthesis of magnesium silicate by precipitation from aqueous solutions of sodium silicate and water-soluble magnesium salts is economical and easy to operate.

\section{Materials and technique}

HTMW method was used to produce magnesium silicate $\mathrm{MgSiO}_{3}$. The synthesis was carried out using Teflon autoclaves in a VOLTA MC-6 multimode microwave oven. The synthesis conditions were as follows: microwave frequency $2.45 \mathrm{GHz}$ (energy $10^{5} \mathrm{eV}$ ), the reaction medium 
temperature 220-240 ${ }^{0} \mathrm{C}$, pressure 27-33 atm, duration 120-180 min. The amounts of the initial solutions were taken to provide a predetermined composition of the final product. The synthesis was carried out by interacting $0,5 \mathrm{M}$ magnesium nitrate solutions with sodium silicate solution $\left(\mathrm{Na}_{2} \mathrm{O} \cdot \mathrm{SiO}_{2}\right)$ keeping the medium $\mathrm{pH}$ in the range of 6.9-7.0. Sodium silicate solutions were obtained by the HTMW method from silica-containing rocks [10-13].

After exposure for a certain time to microwave irradiation, the formed magnesium precipitate was separated by filtration using a Buchner funnel and carefully washed free from $\mathrm{NO}_{3}{ }^{-}$and $\mathrm{Na}^{+}$ ions by hot water, and then dried in a Samsung CE1073AR microwave oven to a moisture content 8-10\%. After drying, the obtained compound was heat-treated in a Nabertherm LHT 08/17 electric furnace. Physicochemical studies of the obtained $\mathrm{MgSiO}_{3}$ silicates were also carried out. Some changes in the physicochemical properties of the samples after their irradiation with fast electrons were determined. Irradiation of samples was performed using ELU-5 linear electron accelerator in the low pressure $\left(10^{-5}-10^{-6} \mathrm{~Pa}\right)$ and temperature $(90 \mathrm{~K})$ mode (the electrons energy $3 \mathrm{MeV}$, dose $10^{16} \mathrm{el} / \mathrm{cm}^{2}$. X-ray phase analysis of the samples was carried out by the powder method using URD63 diffractometer ( $\mathrm{CuK} \alpha$ radiation), and differential thermal and thermogravimetric analysis up to $1500^{\circ} \mathrm{C}$ using STA 449C Jupiter Netzsch (Selb, Germany). The diffuse reflectance of irradiated and nonirradiated samples was determined using FMSh-56M and SPECORD-M-40-UV VIS spectrophotometers. The luminescent properties of the samples were studied using the LUMEN experimental setup. The spectroscopic characteristics (luminescence excitation and luminescence properties) of the samples were studied in a wide spectral range. The experiments were carried out at $300 \mathrm{~K}$ with excitation by UV photons in the ultraviolet and visible spectral regions. As a light source, DKsEL 1000-5 ultrahigh-pressure xenon lamp was used. A highaperture illuminator with optical lenses made of quartz glass was specially manufactured for the xenon lamp. The xenon lamp radiation spectrum power from $240 \mathrm{~nm}$ to $360 \mathrm{~nm}$ was 1000 kilowatts. The samples under study were placed in a process chamber on a copper crystal holder of a blow-off nitrogen cryostat, which provided a high vacuum no less than $2 \cdot 10^{-6}$ Torr, which guaranteed surface cleanliness at low-temperature experiments. The registration system made it possible to measure the luminescence spectra at various spectral points with selective photoexcitation up to $3.5 \mathrm{eV}$, as well as the luminescence spectra in the range from 1.2 to $3.5 \mathrm{eV}$.

\section{Results and discussion}

According to the results of physical-chemical analysis, the synthesized substances represent $\mathrm{MgO} \cdot \mathrm{SiO}_{2} \cdot \mathrm{mH}_{2} \mathrm{O}$ silicate composition: white fine amorphous matter. To determine the temperature of enstatite formation, the obtained $\mathrm{MgSiO}_{3} \cdot \mathrm{mH}_{2} \mathrm{O}$ was heated to $1500{ }^{0} \mathrm{C}$. Fig. 1 presents the results of thermal analysis.

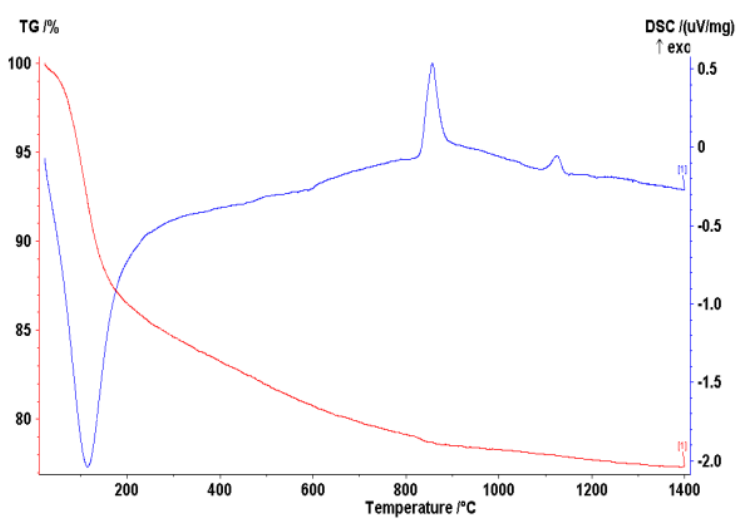

Fig. 1. $\mathrm{MgSiO}_{3} \cdot \mathrm{mH}_{2} \mathrm{O}$ hydrosilicate thermograph. 
The differential heating curve of the $\mathrm{MgSiO}_{3} \cdot \mathrm{mH}_{2} \mathrm{O}$ sample indicates the presence of an exothermic effect within the temperature range of $830-870{ }^{0} \mathrm{C}$ with a maximum at $850{ }^{0} \mathrm{C}$ that corresponds to the transition of the amorphous magnesium metasilicate to crystalline form with the formation of a crystalline phase of enstatite $\left(\mathrm{MgSiO}_{3}\right)$, see Fig. 1. The exothermic effect at $1125{ }^{0} \mathrm{C}$ is also in line with the enstatite crystalline phase that becomes forsterite $\left(\mathrm{Mg}_{2} \mathrm{SiO}_{4}\right)$ at $1500{ }^{0} \mathrm{C}$. This was determined by $\mathrm{X}$-ray analysis of a $\mathrm{MgSiO}_{3}$ sample kept at $850{ }^{\circ} \mathrm{C}$ for 2 hours (see Fig. 2). Up to $1500{ }^{0} \mathrm{C}$, the weight loss was 20-22\%. Apparently, water in the silicate structure is presented in the form of hydroxyl groups, and when heated, a gradual removal of hydroxyl (chemically bound) water takes place.

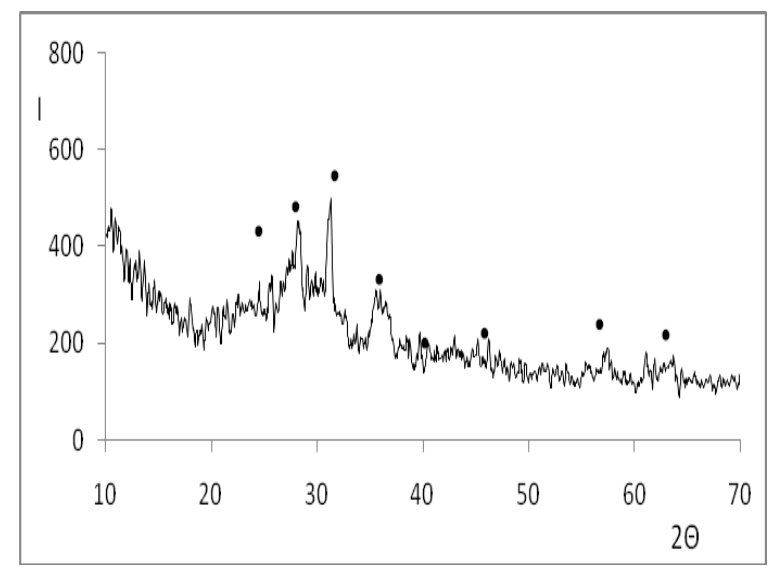

Fig. 2. X-ray diffraction pattern of $\mathrm{MgSiO}_{3} \mathrm{kept}$ at $850{ }^{\circ} \mathrm{C}$ for 2 hours (•-enstatite).

Our studies have shown that after heat treatment at $850{ }^{\circ} \mathrm{C}$, enstatite is the main crystalline phase. The formation of enstatite occurs at a lower temperature (decrease of the enstatite formation temperature makes $200-300{ }^{\circ} \mathrm{C}$ ) and is $2-3$ times faster than the traditional solid-phase methods of its synthesis. Hence, enstatite is formed as early as $850{ }^{\circ} \mathrm{C}$. The microwave synthesis yields better results than traditional methods. This is due to the simultaneous formation of active centers throughout the whole volume of the reaction mixture owing to uniform gradientless heating of the reaction zone by microwave radiation and, in all probability, a local pressure increase in micropores of the resulting product.

Diffuse reflectance of the prepared samples was measured before and after radiation. Fig. 3 presents the obtained results.

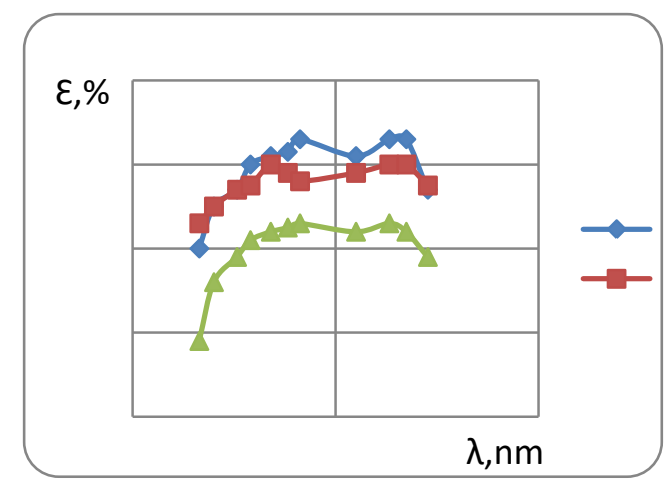

Fig. 3. Diffusion reflection spectra $(\mathcal{E})$ of $\mathrm{MgSiO}_{3}$ samples (MW synthesis at $240{ }^{\circ} \mathrm{C}$ for $1.5 \mathrm{hr}$ ): 1-before irradiation, 2-heat treatment at $850{ }^{\circ} \mathrm{C}$ before irradiation, 3-heat treatment at $850{ }^{\circ} \mathrm{C}$ after $3 \mathrm{MeV}$ electron irradiation. 
It is seen that the enstatite diffuse reflectance is higher, and the decrease in diffusion reflection after the radiation equivalent to 15 -year exposure dose in space, is $10-15 \%$ that is within the allowable rate $(20 \%)$.

The photoluminescence spectra along with the diffuse reflection spectra are sensitive methods for the analysis of powders defectiveness and stability to the action of quanta and charged particles. Therefore, the study of $\mathrm{MgSiO}_{3}$ luminescence spectra in their initial state and after irradiation contributes to the understanding of the regularities of radiation, reflection, and absorption of electromagnetic radiation of the initial and irradiated powders. Changes in the luminescence bands parameters indicate the crystal structure defectiveness.

Fig. 4 shows photoluminescence (PL) spectra of magnesium silicate before and after excitation of luminescence with laser radiation $(405 \mathrm{~nm})$. The results indicate that the luminescence intensity of magnesium silicate increases after radiation.

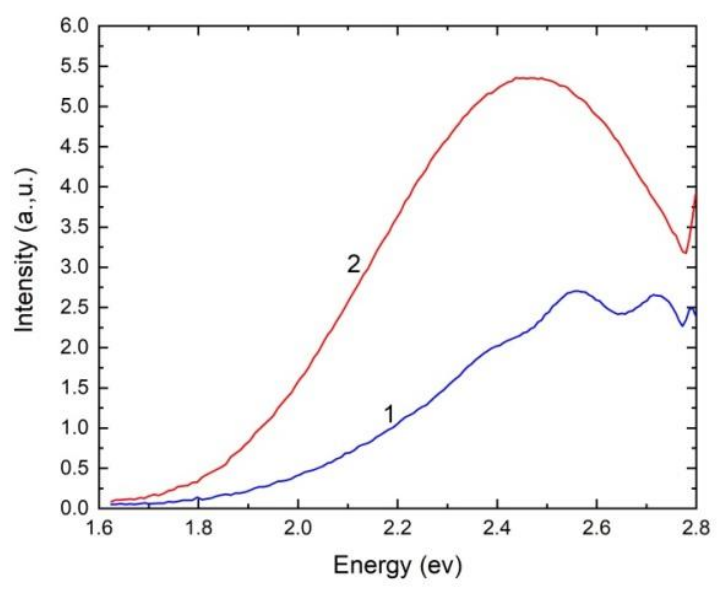

Fig. 4. Photoluminescence spectra of $\mathrm{MgSiO}_{3}$ (MW synthesis at $240{ }^{\circ} \mathrm{C}$ for $1.5 \mathrm{hr}$ ). Excitation by monochromatic laser radiation with a wavelength of $405 \mathrm{~nm}$ at a temperature of $300 \mathrm{~K}$ : 1-unirradiated sample, 2-irradiated with $3 \mathrm{MeV}$ electrons at a dose of $10^{16} \mathrm{el} / \mathrm{cm}^{2}$.

Analysis of the results using the known data on the nature of emission and absorption bands of silicate coatings allows proposing the following interpretation.

In PL spectra, peaks at $2.45 \mathrm{eV}, 2.55 \mathrm{eV}, 2.75 \mathrm{eV}$ can be explained by the presence of multicomponent defects of absorption and radiation centers in the structure of the sample.

Some defect formation mechanisms after electron irradiation of silicates modified by thermal processing or charged particles are known [18-20]: The impact of electrons leads to the formation of interstitial ions of magnesium, oxygen, and their vacancies in various charge states through the following two mechanisms: direct displacement due to elastic interaction; displacement due to ionization and distortion of the crystal lattice electric field. Magnesium ions in various charge states can accumulate in the surface layer, and oxygen ions after the interaction and neutralization pass partially to the vacuum volume. Vacancies of magnesium and oxygen are formed. Numerous knocked-out atoms in the course of these processes produce a cascade of atomic collisions, and as a result, a non-equilibrium and inhomogeneous distribution of point defects is created: vacancies prevail in the center, and interstitial atoms at the periphery [18-20]. The resulting defects drain to the surface where they recombine, therefore without forming additional absorption centers. The high efficiency of the process is determined by the large specific surface area and the energy at which a significant part of collisions occurs in this surface layer.

In the case of irradiation with $3 \mathrm{MeV}$ electrons, the processes of elastic collisions of electrons and nuclei are not typical for such low energies, therefore energy loss is determined by the 
formation of electron-hole pairs $\left(\mathrm{Mg}^{+}, \mathrm{O}^{-}\right)$, spatial separation of which leads to the crystal lattice decomposition and formation of the same types of defects, as at the elastic interaction, as well as by the ionization mechanism, when multiple ionization of atoms leads to displacement of ions from their equilibrium centers due to electrostatic repulsion from surrounding ions. The situation changes if the crystal lattice is distorted as a result of heat treatment or radiation exposure, or if it contains initially charged centers that reduce the symmetry of the surroundings. In this case, the interaction time of the ionized atoms increases, which significantly increases the probability of the defects formation.

PL spectra of samples show that when exposed to $405 \mathrm{~nm}$ laser photons, relatively high luminescence in the spectral range of $1.8 \mathrm{eV}-2.75 \mathrm{eV}$ is observed, and the photoluminescence bands maxima are displaced as a result of redistribution of the emission defect centers formed during heat treatment and irradiation (see Fig. 4). The observed in PL spectra $2.45 \mathrm{eV}, 2.75 \mathrm{eV}$ emission bands, which are characteristic of the luminescence of many silicates, are usually associated with the emission of $\left[\mathrm{SiO}_{4}\right]^{4}$ defect centers caused by local distortions of silicon-oxygen tetrahedra.

The photoluminescence bands with maxima of $2.45 \mathrm{eV}, 2.55 \mathrm{eV}, 2.75 \mathrm{eV}$ observed in nonirradiated and electrons irradiated samples can be mainly caused by the occurrence of intrinsic defects: $\mathrm{Mg}^{2}$ and oxygen ions vacancies, respectively. The maxima within $1.8 \mathrm{eV}-2.75 \mathrm{eV}$ after irradiation is due to the intermediate stage of formation of exciton-defect complexes or short-living defects in the form of $\mathrm{V}_{\mathrm{Mg}}{ }^{-}\left(\mathrm{V}_{\mathrm{Ca}}{ }^{-}\right)$vacancies and $\mathrm{Mg}^{+}$interstitial ions formed at bond breaking as optical transitions in the $\mathrm{V}^{-}$center. The obtained results can also be explained as an effect initiated by the Fermi level shift causing changes in the charge state of the centers and the character of their participation in the luminescence process. The observed wide photoluminescence band, judging by its shape, is a superposition of several emission (absorption) bands after electron irradiation, which is confirmed by the results of [18-21]. Therefore, the shape of the contour of the integral luminescence band and the position of its maxima will change as a result of the influence of radiation. The broad emission band in the visible region is probably caused by intrinsic point defects as well as the anion vacancies with different charge states formed as the result of irradiation, interstitial magnesium ions, and excitons self-trapped on defects. Besides, the emission may also be due to the integration of intrinsic defects with $\mathrm{Mg}^{+}-\mathrm{OH}^{-}$type $\mathrm{OH}-$ groups on the surface.

\section{Conclusions}

Based on physicochemical studies, it was revealed that the microwave method for the synthesis of $\mathrm{MgSiO}_{3}$ from solutions of salts of the initial components is economical and promising. When using the microwave method, crystallization of enstatite comes to an end at lower temperatures (by 200-300 ${ }^{\circ} \mathrm{C}$ ) than using traditional methods. The microwave method provides obtaining fine and homogeneous $\mathrm{MgSiO}_{3}$ crystalline powder at lower temperatures and shorter synthesis times. The synthesized enstatite has higher diffusion reflectance, is radiation-resistant, and can be used as a pigment for thermoregulating coatings.

HTMW synthesis of enstatite is more economical due to shorter synthesis times and low energy consumption.

\section{Acknowledgements}

The authors are grateful to Baghramyan V.V, Sargsyan A.A. (Manvelyan Institute of General and Inorganic Chemistry NAS RA) for the provided samples of thermoregulating coatings synthesized by the hydrothermal-microwave method, as well as for the measurements by X-ray diffraction and diffusion spectra. 


\section{References}

[1] G.N. Maslennikova, I.V. Pish, Ceramic pigments (M., LLC RIF "Stroimateriali", 2009).

[2] M.B. Sedelnikova, Technika i Technologia Silikatov 18 (2011) 13.

[3] M.B. Sedelnikova, N.V. Liseenko, V.M. Pogrebenkov, Izvestija TPU, Chemistry 318 (2011) 31.

[4] Kyu-Ri Pyon, Kyong-Sop Han, Byung-Ha Lee, J. Ceramic Processing Research 12 (2011) 279.

[5] J. Alarcon, J. Eur. Ceram. Soc. 20 (2000) 1749.

[6] C. Valentin, M.C. Munoz, J. Alarcon, J. Sol-Gel Sci. Tech. 15 (2000) 221.

[7] G. Del Nero, G. Cappelletti, S. Ardizzone, P. Fermo, S. Gilardoni, J. European Ceramic Society 24 (2004) 3603.

[8] S. S. Berdanosov, Soros Educational Journal 7 (2001)127.

[9] D.L. Rakhmankulov, I.Kh. Bikbulatov, N.S. Ulaev, S.Yu. Shavshukova, Microwave radiation and intensification of chemical processes (M., Chemistry, 2003).

[10] V.V. Bagramyan, A.A. Sargsyan, C. Ponzoni, R. Rosa, C. Leonelli, Theoretical Foundations of Chemical Engineering 49 (2015) 731.

[11] V.V. Baghramyan, A.A. Sargsyan, A.M. Meliksetian, A.A. Kazaryan, C. Leonello, Chemical Journal of Armenia 66 (2013) 49.

[12] V.V. Baghramyan, Proc. III Intern. Conf. on Chemistry and Chemical Technology 1 (2013) 320.

[13] V.V. Bagramyan, A.A. Sargsyan, C. Ponzoni, R. Rosa, K. Leoneli, Journal of Chemical Technology 10 (2014) 585.

[14] V.V. Bagramyan, A.A. Sargsyan, A.A. Kazaryan, R.V. Harutyunyan, G.H. Petrosyan, A.M. Meliksetyan, Chemical Journal of Armenia 66 (2013) 244.

[15] V.V. Baghramyan, Proc. III Intern. Conf. on Chemistry and Chemical Technology 1 (2013) 323.

[16] V.V Bagramyan, R.V. Harutyunyan, A.A. Sargsyan, A.M. Meliksetyan, A.A. Gazaryan, G.H. Petrosyan, C. Leonello, Chemical Journal of Armenia 66 (2013) 578.

[17] V.V. Baghramyan, A.A. Sargsyan, Materials of the IV Intern. Conf. on Chemistry and Chemical Technology 1 (2015) 61.

[18] M.M. Mikhailov, Neorganicheskie Materiali 24 (1988) 415.

[19] M.M. Mikhailov, Prediction of Optical Degradation of Spacecraft Thermoregulating Coatings (Novosibirsk: Nauka, 1999).

[20] M.M. Mikhailov, Photostability of Thermoregulatory Surfaces of Space Vehicles (Tomsk: Publishing house of Tomsk University, 2007).

[21] A.O. Badalyan, Armenian Journal of Physics 14 (2021) 85. 